Check for updates

Cite this: RSC Adv., 2018, 8, 34182

\title{
Electronic and thermal spin effect of molecular nanowires between graphene electrodes
}

\begin{abstract}
X. Q. Deng (D)* and R. Q. Sheng
Based on the first-principles method, the electronic spin transport properties of terphenyl molecule bridging in zigzag graphene nanoribbon (ZGNR) electrodes with three connecting linkages were investigated, including dangling, heptagon, and pentagon-linkages. For the pentagon-linkage system, we observed a perfect spin filtering effect in the parallel (P) configuration (at almost $100 \%$ spin polarization), with the heptagon-linkages system following next (85-95\% spin polarization), however, the spin filtering effect is almost negligible for the dangling-linkages system. In the antiparallel (AP) configuration, the pentagon- and heptagon-linkage systems also showed a high spin filtering effect. The terphenyl molecule was then replaced by carbon chains based on the pentagon-linkages, and these devices also show a perfect spin filtering effect (100\% spin polarization). Finally, the thermally induced spin transport for the carbon chains model with pentagon-linkages was explored, and this system exhibits almost $100 \%$ thermal spin polarization.
\end{abstract}

Received 16th August 2018

Accepted 9th September 2018

DOI: $10.1039 / \mathrm{c} 8 \mathrm{ra06852f}$

rsc.li/rsc-advances

can exhibit a spin polarization, ${ }^{18}$ but the spin-filtering efficiency is less than $80 \%$. When ZGNRs and armchair graphene nanoribbons (AGNR) are connected by pentagon-heptagon (5-7) defect-lines, different spin polarizations can be obtained. More interesting, defect-line types obviously affect the polarization efficiency. ${ }^{19}$ However, the departmental influence of pentagonheptagon (5-7) defects on the spin transport properties are not clear. For single molecules attached between two electrodes, interference effects originating from different anchoring groups or positions clearly affect the transport of electrons. ${ }^{20}$ For example, a polyacetylene chain bridging the graphene sublattice of ZGNRs may lead to two bonding arrangements, and metallic or semiconducting systems can be obtained owing to the local coupling. ${ }^{21}$ Moreover, spin caloritronics are attracting growing interest, as they combine the advantages of thermoelectronics and spintronics with thermally induced spin currents in the absence of an external bias voltage. ${ }^{22,23}$ In this paper, we study the spin transport properties of the terphenyl molecule connected by two ZGNR electrodes with different connecting configurations, which include dangling, ${ }^{24}$ heptagon, ${ }^{19}$ and pentagon-linkages. ${ }^{18}$ For the pentagon-linkages system, we can observe a high spin filtering effect in the parallel (P) configuration, this is followed closely by the heptagonlinkages system, while the spin filtering effect is almost negligible for the dangling-linkages system. In the antiparallel (AP) configuration, the pentagon- and heptagon-linkages systems also show a spin filtering effect. The terphenyl molecules were then replaced by carbon chains based on pentagon-linkages, and these devices were also found to show a perfect spin filtering effect in the $\mathrm{P}$ and AP configurations. Finally, thermally induced spin transport for the carbon chains model with 
pentagon-linkages was explored, and this system exhibits almost a $100 \%$ thermal spin polarization.

\section{Models and methods}

The ribbon widths of ZGNRs can be characterized by the number of zigzag $\mathrm{C}$ chains, $\mathrm{N}$, along the nanoribbon axis, and is denoted as NZGNRs. An even and odd $\mathrm{N}$ correspond to
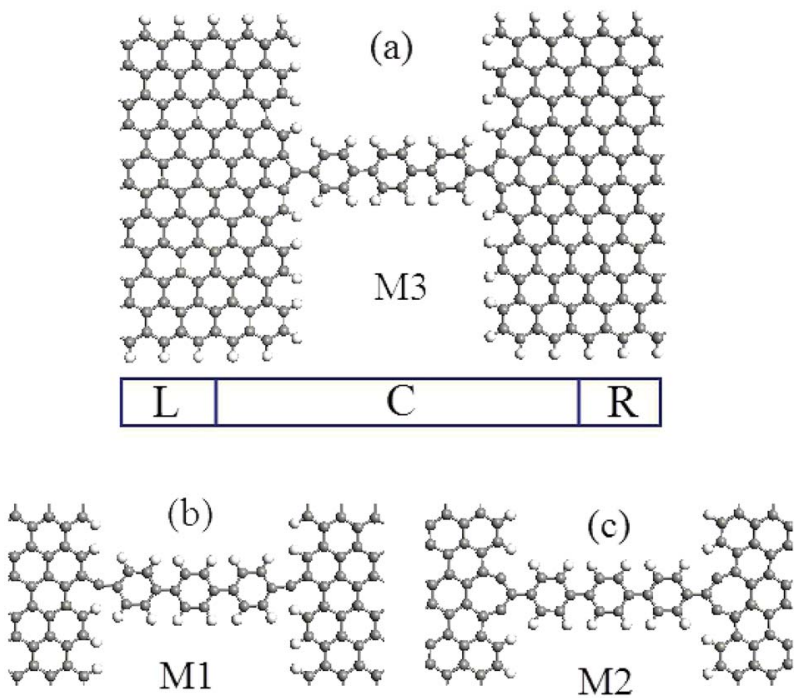

Fig. 1 (a) The geometric structures of model M3, (b) and (c) correspond to the different connection interfaces between the electrodes and the central molecule for models $M 1$ and M2. $L(R)$ and $C$ indicate the left (right) electrodes, and the central scattering region, respectively. symmetric and asymmetric ZGNRs. Here, we chose 11 and 12ZGNRs as the two types of electrodes. The structure of the molecular device is illustrated schematically in Fig. 1, which is divided into three regions, left (right) electrode and the scattering region. For M1 (M2, M3), the connecting configurations between the terphenyl molecule and the ZGNRs electrodes are dangling (pentagon, heptagon).

The geometry optimizations and the calculation of the electronic properties were performed using a first-principles method based on density functional theory (DFT) combined with the non-equilibrium Green's function (NEGF) technique, as implemented in the Atomistix ToolKit software. The spin generalized gradient approximation (SGGA) was used as the exchange-correlation functional. The real space grid techniques were used with an energy cutoff of $200 \mathrm{Ry}$ as the required cutoff energy in numerical integrations and as the solution to the Poisson equation using a fast Fourier transform (FFT). The geometrical structures used were optimized until all residual forces on each atom were smaller than $0.01 \mathrm{eV} \AA^{-1}$ under the periodic boundary condition. The wave function was expanded by a double-zeta plus polarization (DZP) for all atoms. The current, $I$, in the systems, as a function of the applied external bias, $V$, can be calculated from the Landauer-like formula: ${ }^{25}$ $I_{\sigma}=(\mathrm{e} / \mathrm{h}) \int_{-V / 2}^{V / 2} T_{\sigma}(E, V) \mathrm{d} E$, in which $\sigma=\alpha$ and $\beta$ spin. The region of the bias window is $[-\mathrm{eV} / 2,+\mathrm{eV} / 2]$, and $T_{\sigma}(E, V)$ is the bias-dependent transmission coefficient.

\section{Results and discussion}

When the external magnetic fields applied to two electrodes are the same or in the opposite direction, magnetic ordering is denoted as parallel $(\mathrm{P})[1,1]$ or antiparallel (AP) $[1,-1] .^{14}$

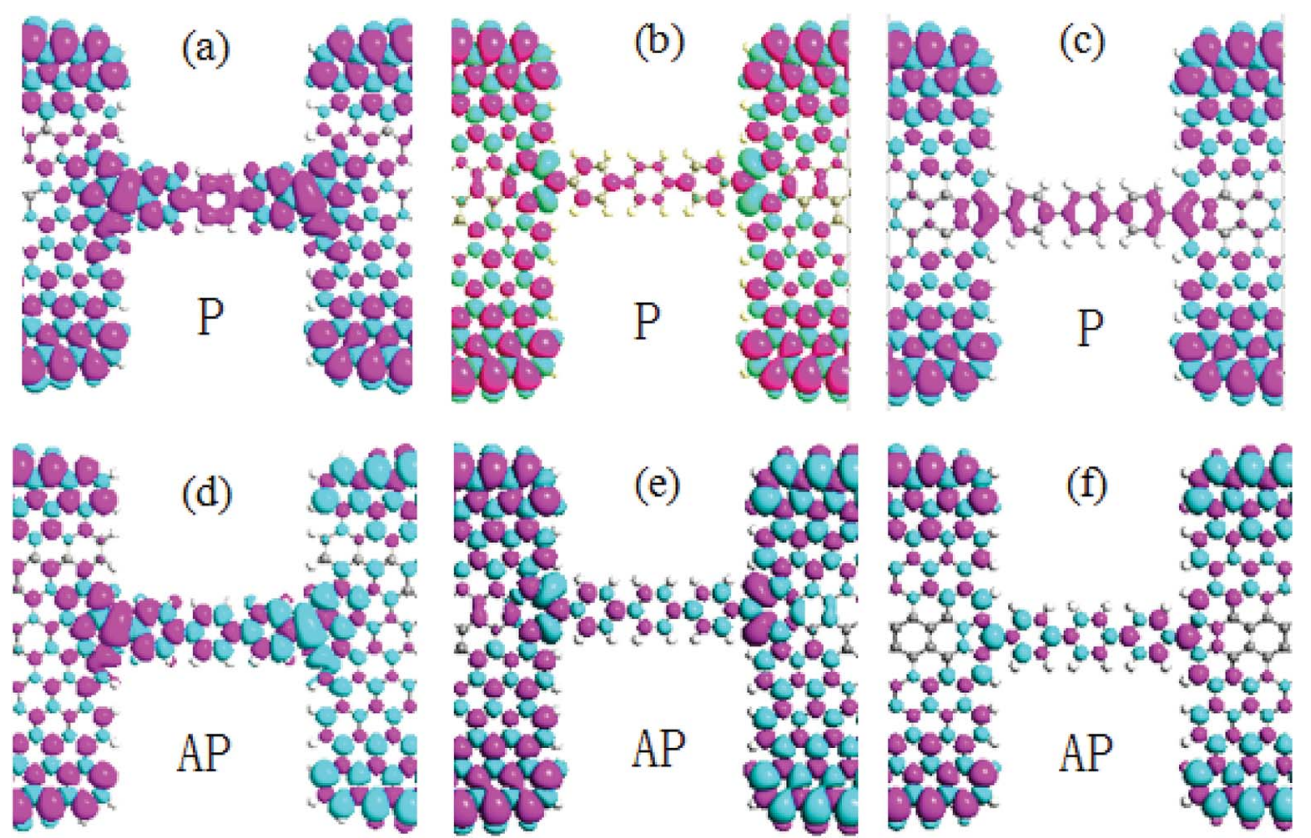

Fig. 2 The spin density isosurfaces for $M 1-M 3$ in the $P$ and $A P$ configurations: (a) $M 1$ in $P$ configuration; (b) $M 2$ in $P$ configuration; (c) $M 3$ in $P$ configuration; (d) M1 in AP configuration; (e) M2 in AP configuration; and (f) M3 in AP configuration. 

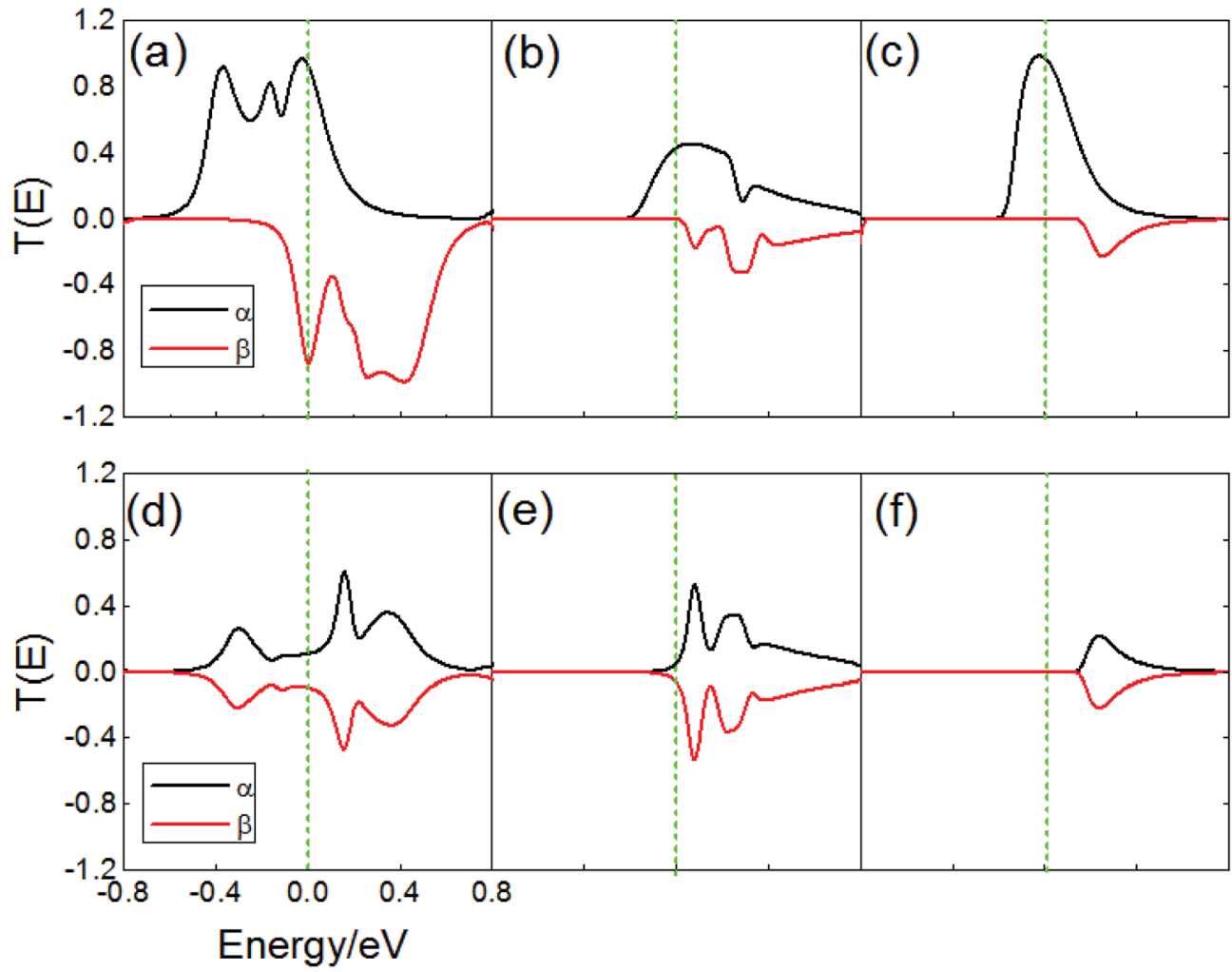

Fig. 3 (a)-(c) Transmission spectra for $M 1-M 3$ in the P and (d)-(f) AP configurations. The Fermi level is set to zero, as indicated by the green vertical dotted lines.

Table 1 The $\alpha$ and $\beta$ LDOS spin density for M1-M3 in the P configuration

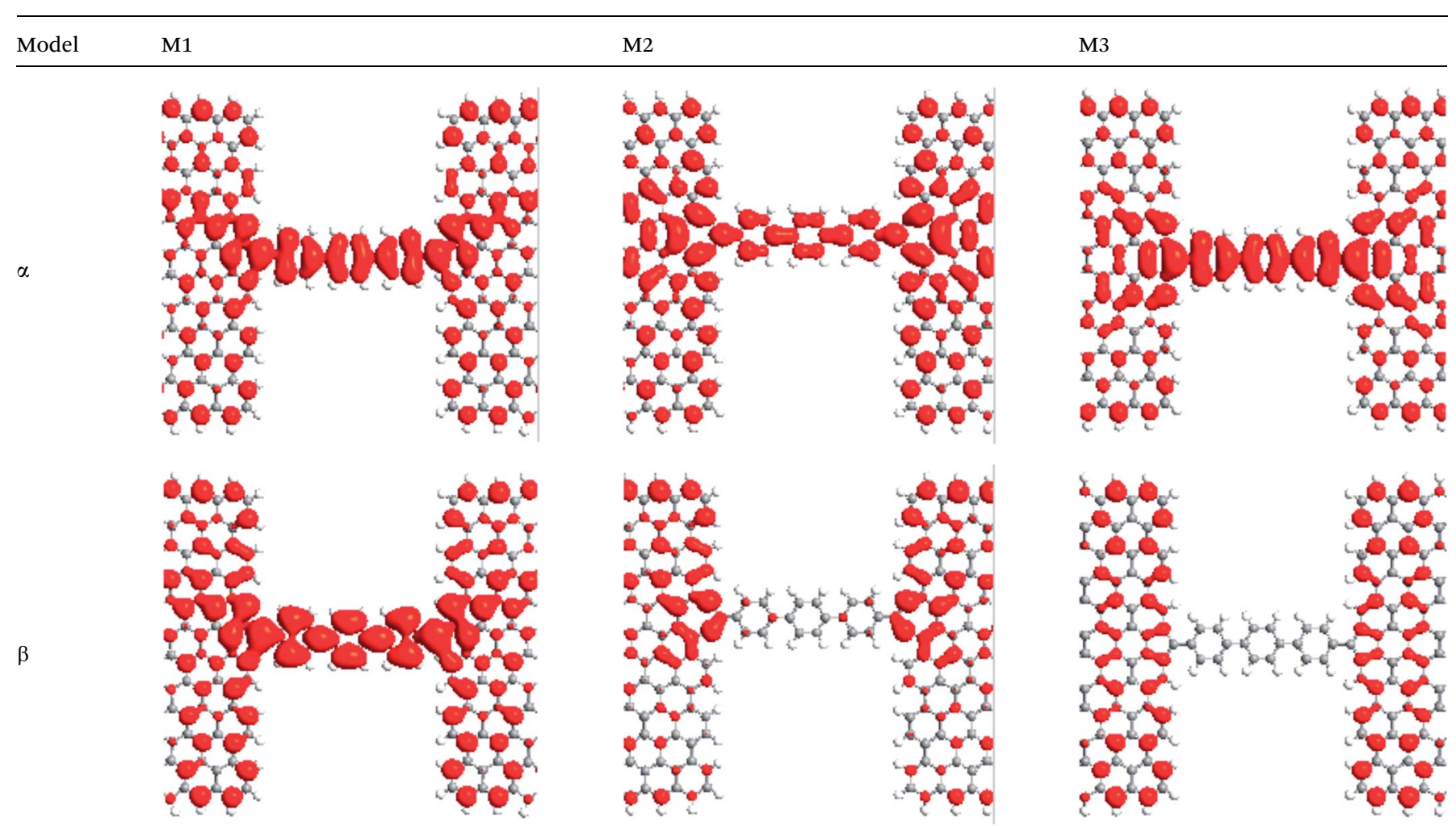



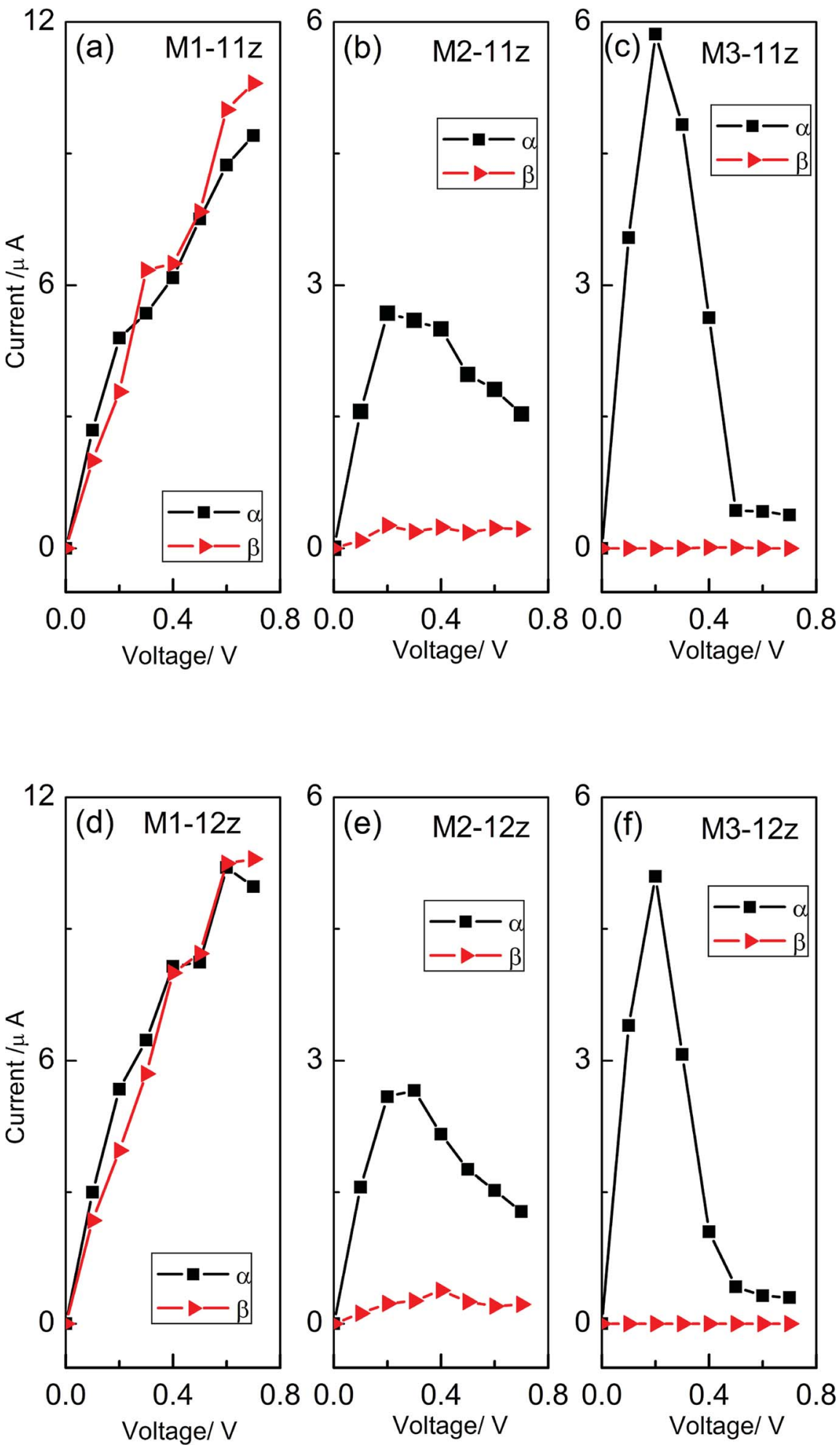

Fig. 4 (a)-(c) The I-V curves for M1-M3 with a ZGNR width of 11 in the P configuration, and (d) - (f) the I-V curves for M1-M3 with a ZGNR width of 12 in the $P$ configuration.

Fig. 2a-f demonstrates the spin density for M1-M3 with the isosurface of the spin density $\left(\nabla \rho=\rho_{\alpha}-\rho_{\beta}\right)$ in the P and AP magnetic configurations, in which $\rho_{\alpha}$ and $\rho_{\beta}$ denote the electron density of the $\alpha$-spin (magenta) and $\beta$-spin (cyan), respectively. As can be seen, the localized magnetic distribution at the interface is obviously different, which is a reduction at the interface from M1 to M2 (M3). Meanwhile, the magnetic distribution for the two spin component in the molecules are completely different in each of the three models, and change with the external magnetic configurations. Using M3 as an 


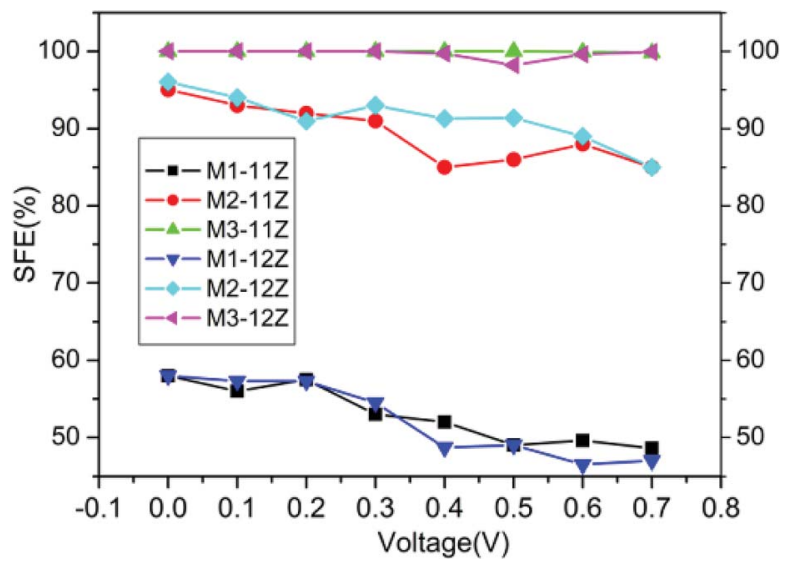

Fig. 5 The SFE curves for M1-M3 with different ZGNR widths of 11 and 12 in the $\mathrm{P}$ configuration.

example, there exists a net $\alpha$-spin magnetic moment at the molecule and the interface with $\mathrm{M} 3$ in the $\mathrm{P}$ configuration, while both the $\alpha$ - and $\beta$-spin magnetic moment are distributed asymmetrically on the molecule in the AP configuration, as shown in Fig. $2 \mathrm{c}$ and $\mathrm{f}$, respectively, which give rise to a spin splitting and degeneration in the central region. Fig. 3 shows the transmission spectra for M1-M3 at zero bias in the P and AP configurations, which show completely different behaviors at equilibrium. It is notable that the transmissions are different: for M1, the transmission coefficients are finite at the Fermi level for two spins, and the value in the $\mathrm{P}$ configuration is larger than the AP configurations (Fig. 3a and d). While the $\beta$-spin transmission coefficients are almost equal to zero at the Fermi level for M2 (M3) in the P configuration, and pronounced $\alpha$-spin transmission peaks can be observed in the Fermi level region, which contributes to the $\alpha$-spin current. Therefore, the spinfilter efficiency (SFE) is $\eta=\mid\left(T_{\alpha}-T_{\beta}\right) /\left(T_{\alpha}+T_{\beta}\right),{ }^{26}$ and $100 \%$ spin polarization can be obtained. For M3, the energy position of the $\beta$-spin transmission peak is far away from the Fermi level when compared with M2, we can predict that M3 will show a larger bias region with a spin filtering effect. For the AP state, the three models show spin-degenerate behaviors, which originate from the mismatch of the scattering states between the two electrodes with opposite spin directions.

To provide an insight into the transmission behavior discussed above, we investigated the local density of states (LDOS) at the Fermi level in the P configuration, which can describe the space-resolved density of states (DOS). ${ }^{27,28}$ The LDOS can tell us the contributions of every atom to the device DOS, and can be used to gain insights into a solid-state device. From Table 1, it is evident that the LDOS of the $\alpha$ - and $\beta$-spin for M1 are delocalized on the terphenyl molecules, interfaces and two electrodes. There are large overlaps between the extended $\pi$-orbitals of the dangling carbon atoms and the delocalized big $\pi$-orbital which origin from the terphenyl ring and electrodes. This is to say, $\alpha$ - and $\beta$-spin scattering states are well extended on the whole on the devices. Thus, the two kinds of spin electrons can easily transport from one electrode to the other one. For M2 (M3), the LDOS of $\alpha$-spin are completely delocalized on the whole device, and extended orbitals are formed between the pentagon (heptagon) and terphenyl molecule, and the electrons can easily go through the devices. The LDOS of $\beta$-spin for M2 and M3, however, are localized mainly on the electrodes, and
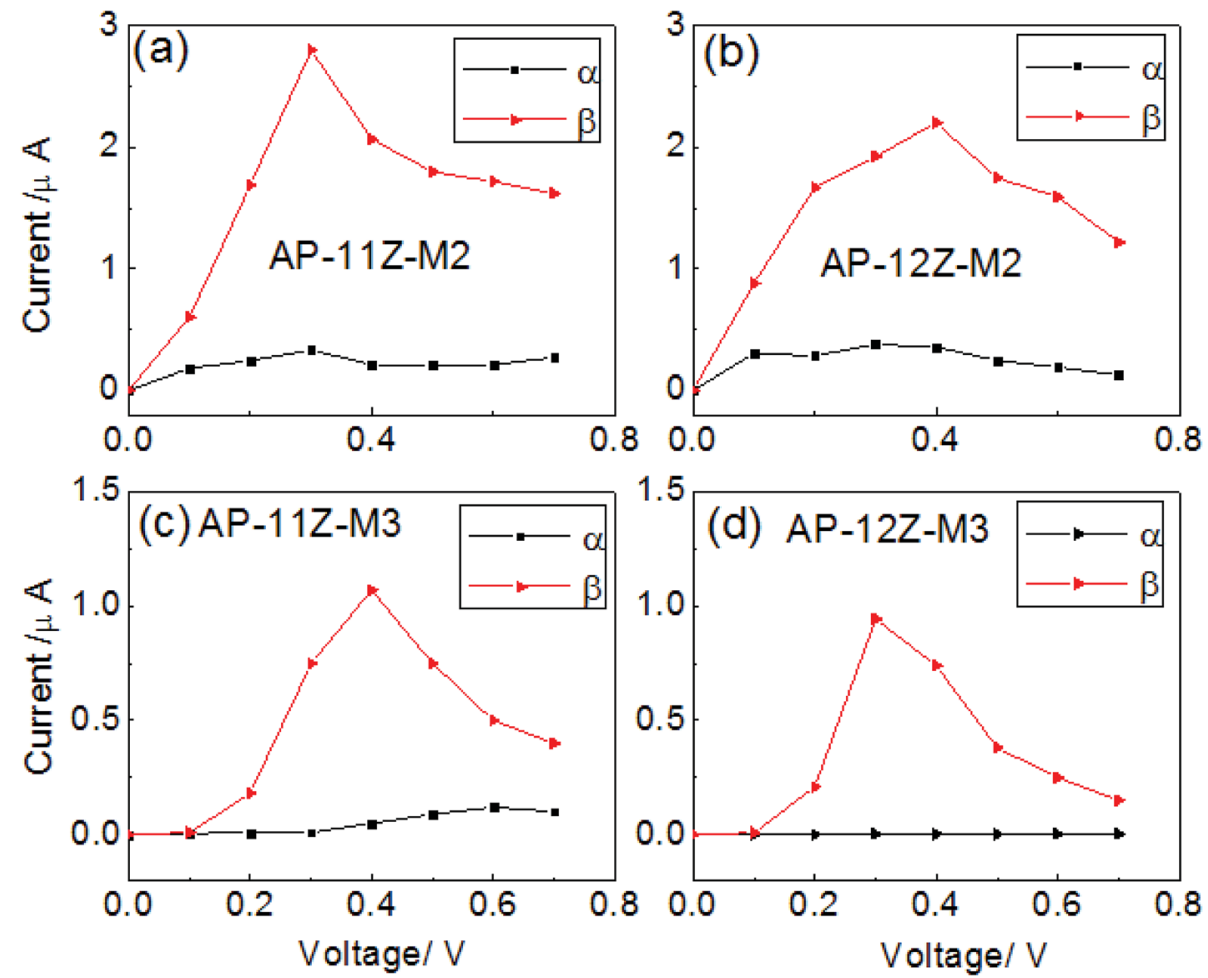

Fig. 6 The I-V curves for M2 and M3 with different ZGNR widths 11 and 12 in the AP configuration: (a) M2 11; (b) M3 11; (c) M2 12; and (d) M3 12. 

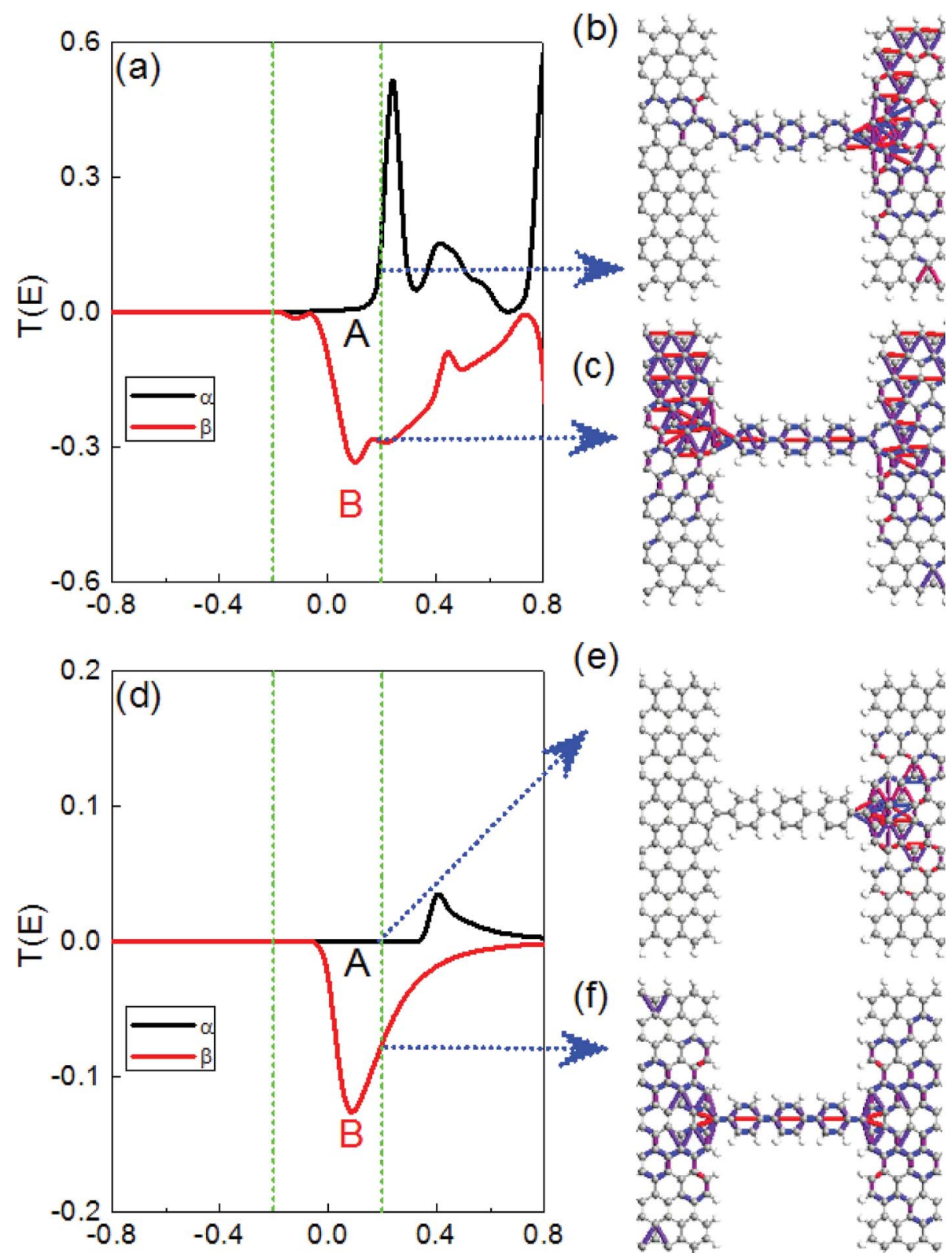

e)

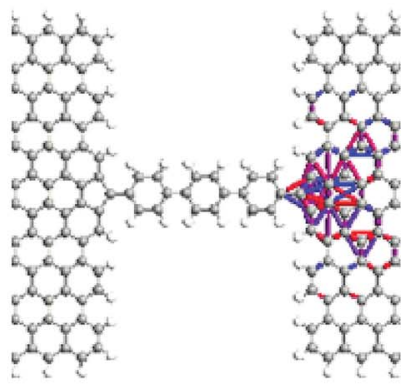

(f)

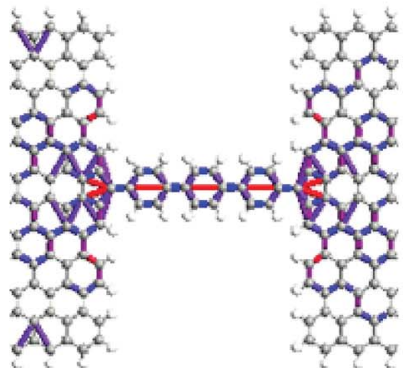

Fig. 7 (a) and (d) transmission spectra for M2 and M3 with a ZGNR width of 12 in the AP configuration at a $0.4 \mathrm{~V}$ bias, (b) and (e) correspond to $\alpha$ spin transmission pathways, (c) and (f) correspond to $\beta$-spin transmission pathways, the energy point is $0.2 \mathrm{eV}$, the region between the two green dashed lines indicates the bias window, and the Fermi level is set to zero.

rarely on the terphenyl molecules. As a result, the $\beta$-spin transmission coefficients near the Fermi level are about zero.

Fig. 4 displays the spin-resolved $I-V$ characteristics for M1M3 with ZGNR widths of 11 and 12 in the P configuration. For convenience, we marked them as $\mathrm{M} 1(2,3)-\mathrm{NZ}$, then in the AP configurations, we marked them as AP-M1(2,3)-NZ, N $=11$ and 12. As can be seen, they are obviously different. This indicates that the magnetic transport feature strongly depends on the combined manner and is independent of the ZGNR widths. For M1, both the $\alpha$ - and $\beta$-spin currents rise almost monotonously when the bias is increased, as shown in Fig. $4 \mathrm{a}$ and d. While for M2, $I_{\alpha}$ increases linearly when the bias voltage increases from zero to about $0.3 \mathrm{~V}$ and then decrease gradually, which is a negative differential resistance (NDR), and $I_{\beta}$ shows the familiar law, but its value is much less than $I_{\alpha}$ for the entire bias voltages, as shown in Fig. $4 \mathrm{~b}$ and e. For M3, $I_{\alpha}$ also shows NDR behavior and reaches a maximum value at $0.2 \mathrm{~V}$, but $I_{\beta}$ is always negligibly small in the calculated bias region, almost entirely suppressed, as shown in Fig. $4 \mathrm{c}$ and f. Therefore, the pentagon system permits $\alpha$-spin electrons transmission but scatters $\beta$ spin electrons completely. However, a perfect ZGNR device does not show any spin polarization under the $\mathrm{P}$ configuration. ${ }^{14,29}$ Therefore, a remarkable rise in the spin polarization for $\mathrm{M} 2$ and $\mathrm{M} 3$, and an interface-induced special spin polarization can be obtained. ${ }^{30,31}$ Here, we define the SFE as $\eta=\mid\left(I_{\alpha}-I_{\beta}\right) /\left(I_{\alpha}+I_{\beta}\right)$. The calculated results for the magnetic device effects are plotted in Fig. 5. Obviously, in the P configuration, M3 exhibits a spin filtering effect with a high SFE approaching almost $100 \%$ in 

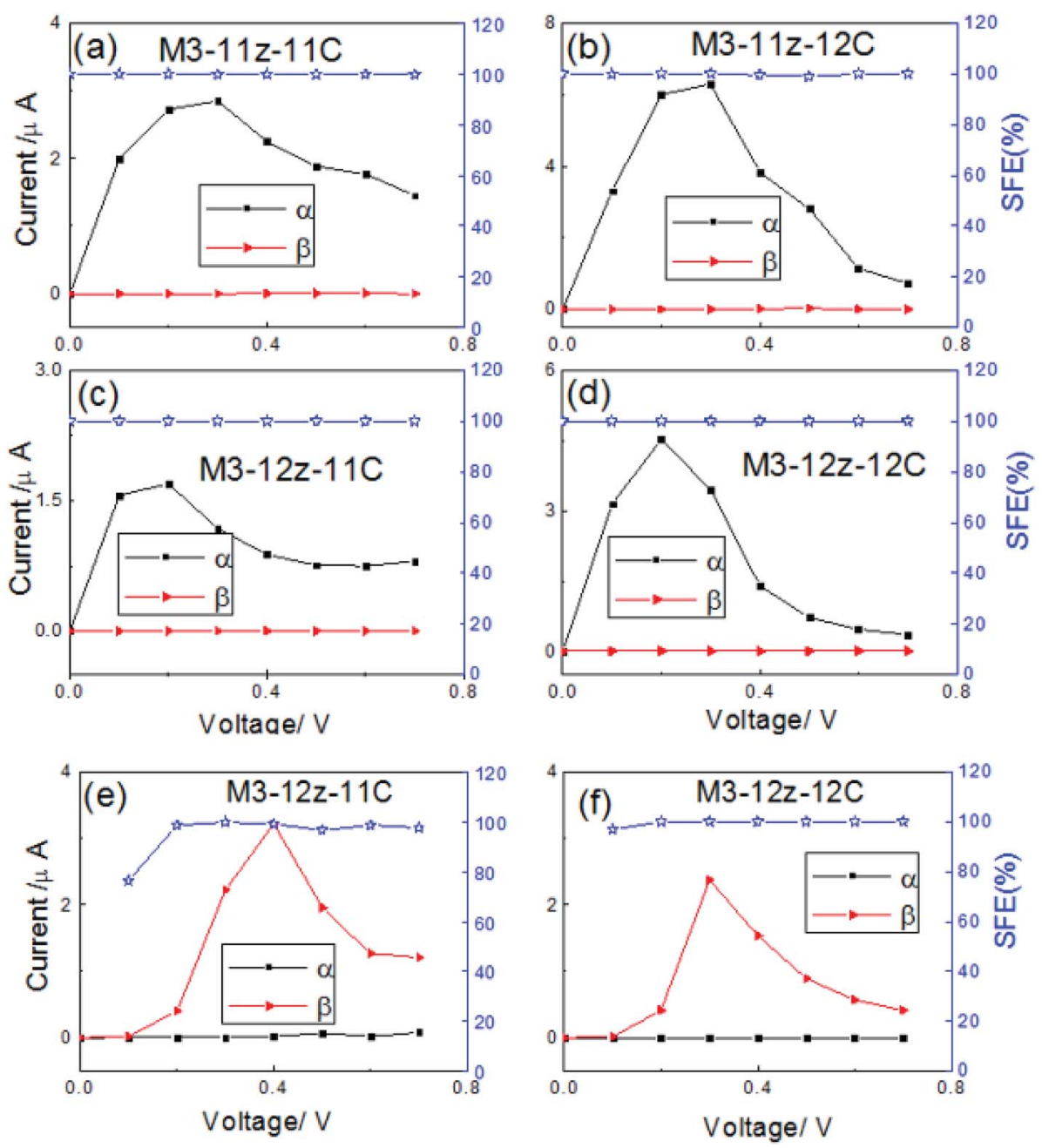

Fig. 8 (a) - (d) The $I-V$ and SFE curves for the carbon chain models with ZGNR widths of 11 and 12 in the P configuration, (e) and (f) are in the AP configuration with $12 Z G N R$, the connection interface is a pentagon.

a very large bias region from 0 to $0.7 \mathrm{~V}$. The $\mathrm{SFE}$ of $\mathrm{M} 2$ is from $85 \%$ to $95 \%$ in the whole bias region. On the other hand, the SFE of M1 can be neglected. This suggests that the pentagon structure is favorable for spin filtering of the electron. In the AP configuration, the case is different, as shown in Fig. 6. As an example, M3 has a large $I_{\beta}$ except at $0.1 \mathrm{~V}$, and a negligible small $I_{\alpha}$ at the bias, which shows the higher spin filtering effect. Due to the existence of the threshold voltage at $0.1 \mathrm{~V}$, the currents in the AP configuration are lower than in the P configuration. M2 also shows this spin polarization feature, but the SFE is still lower than M3. In other words, the perfect spin filtering effect can be obtained in the $\mathrm{P}$ and AP configurations.

To give a better explanation of the spin filtering effect, the spin-dependent transmission spectra for M2 and M3 at $0.4 \mathrm{~V}$ bias were plotted and are shown in Fig. $7 \mathrm{a}$ and d. The transmission spectrum of the two spin channels are obviously different. High $\beta$-spin transmission peaks for the two models are observed within the bias windows, which are $[-0.2,0.2] \mathrm{eV}$. In contrast, the $\alpha$-spin transmission coefficients remain at zero in the bias windows for $\mathrm{M} 3$, while there is a tail existence of the high $\alpha$-spin peak near the $0.2 \mathrm{eV}$ energy for M2. Therefore, the $\beta$ - spin current is much larger than the $\alpha$-spin, leading to a spin filtering effect, and M3 shows a higher perfect SFE than M2. Next, we investigated the electron transmission pathway, which can show where (and how) the current propagates. ${ }^{32-34}$

The $\alpha$ - and $\beta$-spin transmission pathway at $0.2 \mathrm{eV}$ for M2 (M3) are shown in Fig. 7b, c, e, and f. Two different current channels exist, one via a chemical bond and one obtained through hopping between atoms. It is notable that the $\alpha$ - and $\beta$ spin electron for M2 can flow through the central molecule mainly via a chemical bond and can reach the other electrode through two current channels, as well as the $\beta$ electron of M3. However, the $\alpha$ electron cannot transmit on the device, and the corresponding transmission is weak for M3, as shown in Fig. 7e.

Next, the terphenyl molecules were replaced by the carbon chains based on M3. Here, the width of ZGNR is $\mathrm{N}=11$ and 12 . The carbon chain with odd (even) carbon atoms shows a different bond type, we chose two kinds of carbon chain consisting of 11(12) carbon atoms. Considering the electrode and the length of the carbon chains, we designed four models, denoted as M3-11z-11(12)C and M3-12z-11(12)C. The spin current-voltage $(V)$ characteristics of the four models are 

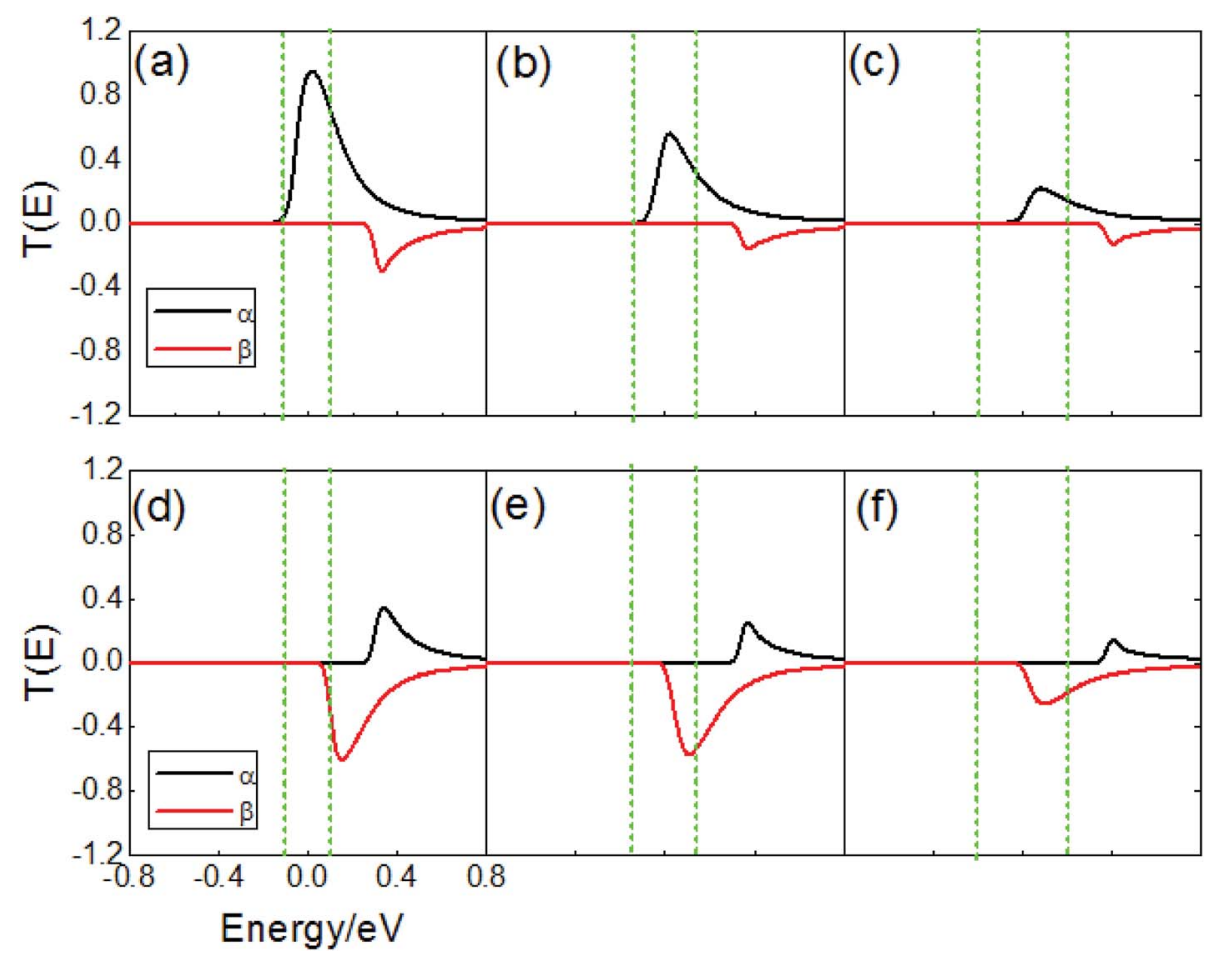

Fig. 9 (a)-(c) Transmission spectra of $M 3-12 Z-12 C$ at $0.2-0.4 \mathrm{~V}$ in the $\mathrm{P}$ magnetism configuration, and (d) $-(\mathrm{f})$ in the $A P$ magnetism configuration.

presented in Fig. 8a-d. One can see that the $\alpha$-spin current increases obviously from the 0 to $0.2 \mathrm{~V}(0.3 \mathrm{~V})$ bias, then decreases gradually, the currents for the models of the 12 carbon chain drop more quickly than those for the 11 carbon chain, and the NDR can be obtained for these models. However, the $\beta$-spin current is always negligibly small within the whole bias range. This suggests that the $\alpha$-spin electrons can flow through the carbon chain freely, but that the $\beta$-spin electrons are scattered completely, which shows a perfect spin filtering behavior. In the AP configuration, using M3-12Z-11(12)C as an example, the $\alpha$-spin currents are almost zero in the whole bias region, but the $\beta$-spin currents show a high value after $0.2 \mathrm{~V}$, as shown in Fig. 8e and $\mathrm{f}$. It is thus clear that a perfect spin filtering effect (around $100 \%$ SFE) can be realized in the AP configuration. In order to explain the spin $I-V$ characteristics of devices for M3-12Z-12C, we created a comparison chart for the transmission spectra at various bias in Fig. 9. In the P configuration, a typical resonant tunneling of $\alpha$-spin electrons in the vicinity of Fermi level are found, which weaken with increasing bias, leading to a decrease in the $\alpha$-spin currents, and the $\beta$-spin transmission channel cannot enter into the bias windows, therefore, a perfect spin-filtering effect can be obtained. In the AP configuration, the $\alpha$-spin transmission peak shifts to a high energy and always stays outside of the bias windows, and the current is determined by the $\beta$-spin tunneling integral within the bias window, which arrives at the maximum value at a bias of $0.4 \mathrm{~V}$.

Finally, the spin-dependent thermoelectronic transport calculations were also investigated for $\mathrm{M} 3-12 \mathrm{Z}-12 \mathrm{C}$ in the $\mathrm{P}$ magnetism configuration as an example of the pentagon- linkage system. The thermally-driven spin-dependent current through the device is calculated using the Landauer formula: $I_{\sigma}=(\mathrm{e} / \mathrm{h}) \int_{-\infty}^{\infty} T_{\sigma}(E)\left[f_{\mathrm{L}}\left(E, T_{\mathrm{L}}\right)-f_{\mathrm{R}}\left(E, T_{\mathrm{R}}\right)\right] \mathrm{d} E$, in which $\sigma$ is the index of the $\alpha$ - and $\beta$-spin, $T_{\mathrm{L}}$ and $T_{\mathrm{R}}$ represent the temperature of the left and right electrode. The difference in the carrier concentrations between the left and the right electrode is determined by the Fermi distribution $\left(f_{\mathrm{L}}\left(E, T_{\mathrm{L}}\right)-f_{\mathrm{R}}\left(E, T_{\mathrm{R}}\right)\right)$, which is intimately related to the electron temperature at the two electrodes. ${ }^{35}$ Fig. 10a shows the spin-dependent currents versus the temperature difference $\left(\Delta T=T_{\mathrm{L}}-T_{\mathrm{R}}\right)$, in which $T_{\mathrm{L}}=$ $200(300,400) \mathrm{K}$. As can be seen from the figure, the $\alpha$-spin current can pass through the device, which increases linearly with the increase of $\Delta \mathrm{T}$, and the $\beta$-spin current is strongly suppressed. As $I_{\alpha}$ is always much larger than $I_{\beta}$ at the same $\Delta \mathrm{T}$ and $T_{\mathrm{L}}$, a perfect thermal spin-filtering effect can be obtained, and the polarization of the spin current is close to $100 \%$. The thermally-driven spin currents through the device are the joint outcomes of the transmission spectra and the difference in the Fermi-Dirac distributions. Owing to the exponential decaying nature of the Fermi distribution, the thermally-driven currents only depend on the transmission near the Fermi energy. The $\alpha$ spin current spectra $J(E)=T(E)\left[f_{\mathrm{L}}\left(E, T_{\mathrm{L}}\right)-f_{\mathrm{R}}\left(E, T_{\mathrm{R}}\right)\right]^{35}$ is plotted in Fig. 10b, in which the cover area under the curves determines the thermally-driven current and $\Delta T=60 \mathrm{~K}$ with different $T_{\mathrm{L}}=$ 200,300 , and $400 \mathrm{~K}$. The cover area of the $\alpha$-spin $J(E)$ above the Fermi level is larger than that below the Fermi level at fixed $T_{\mathrm{L}}$, resulting in the hole current $I_{\mathrm{h}}$ being larger than the electron current $I_{\mathrm{e}}$. Therefore, a positive $\alpha$-spin current can be obtained. For $\beta$-spin, $J(E)$ almost disappears for different $T_{\mathrm{L}}$ owing to there being no $\beta$-spin transmission close to the Fermi energy. 

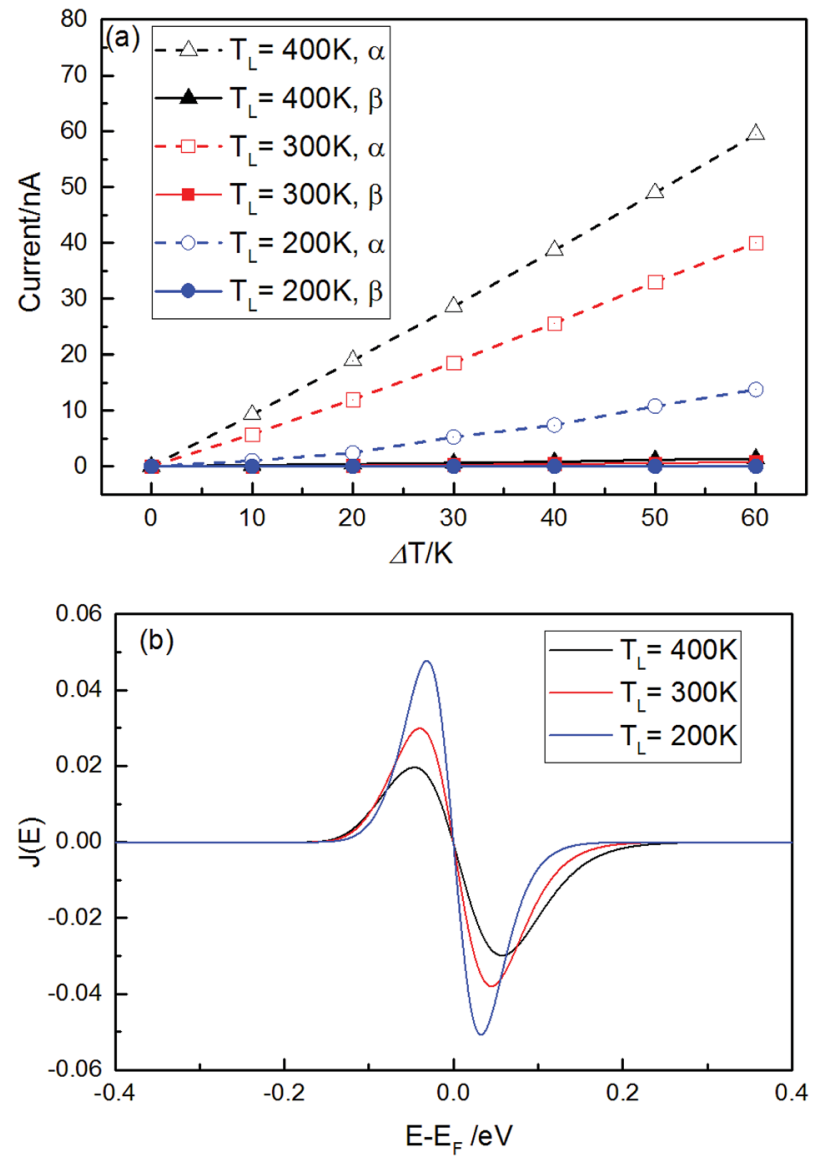

Fig. 10 (a) The spin dependent currents versus $\Delta T$ for different $T_{L}$. (b) The $\alpha$-spin current spectra for different $T_{\mathrm{L}}\left(T_{\mathrm{L}-\mathrm{R}}=60 \mathrm{~K}\right)$.

\section{Conclusions}

Based on the NEGF method combined with DFT, we investigated the spin transport properties for terphenyl molecule bridging in ZGNR electrodes with different connecting configurations, which included dangling, heptagon, and pentagon-linkages. The results show that the transport properties are affected by the connecting configurations and spin configuration of the electrodes. For the pentagon-linkages system in the $\mathrm{P}$ configuration, a very high spin filtering effect (close to $100 \%$ spin polarization) can be obtained, followed by the heptagon-linkages system, and the spin filtering effect of the dangling-linkages system is almost negligible. In the AP configuration, however, it is interesting that the pentagonand heptagon-linkages systems show an adverse spin filtering effect compared with the $\mathrm{P}$ configuration. We also investigated the spin transport properties of the carbon chains connected with ZGNR electrodes with pentagon-linkages in the P and AP configuration, and found that the spin filtering effects are intrinsic. Finally, we further investigated the spin-dependent thermoelectronic transport properties of the carbon chains model with pentagon-linkages system, and the results show that this system exhibits almost $100 \%$ thermal spin polarization. The obtained results will provide structural models, theoretical references, performance parameters, and new ideas for the delicate manufacture of functional magnetic devices.

\section{Conflicts of interest}

There are no conflicts to declare.

\section{Acknowledgements}

This work was supported by the National Natural Science Foundation of China (Grant No. 61771076, 61371065 and 51604042), the Aid Program for Science and Technology Innovative Research Team in Higher Educational Institutions of Hunan Province, and the Scientific Research Innovation Fund for Postgraduate of Changsha University of Science and Technology.

\section{References}

1 X. K. Chen, J. Liu, Z. H. Peng, D. Du and K. Q. Chen, Appl. Phys. Lett., 2017, 110, 1121.

2 T. Low and F. Guinea, Nano Lett., 2010, 10, 3551.

3 J. M. Zheng, P. Guo, Z. Ren, Z. Jiang, J. Bai and Z. Zhang, Appl. Phys. Lett., 2012, 101, 666.

4 Q. X. Dong, R. Hu, Z. Q. Fan and Z. H. Zhang, Carbon, 2018, 130, 206-214.

5 X. Q. Deng, Z. H. Zhang, G. P. Tang, Z. Q. Fan, C. H. Yang and L. Sun, Carbon, 2014, 66, 646-653.

6 W. Han and R. K. Kawakami, Phys. Rev. Lett., 2011, 107, 047207.

7 X. Q. Deng, Z. H. Zhang, G. P. Tang, Z. Q. Fan, C. H. Yang and L. Sun, Carbon, 2015, 94, 317-325.

8 R. R. Pandey, M. Fukumori, A. Termeh, M. Eguchi, D. Tanaka, T. Ogawa and H. Tanaka, Nanotechnology, 2017, 28, 175704.

9 K. Janani and D. J. Thiruvadigal, Appl. Surf. Sci., 2018, 449, 829-837.

10 L. Miao, R. Jia, Y. Wang, C. P. Kong, J. wang, R. Eglitis and H. X. Zhang, J. Saudi Chem. Soc., 2017, 21, 111-117.

11 Y. An and Z. Yang, Appl. Phys. Lett., 2011, 99, 192102.

12 H. S. Moon, J. M. Yun, K. H. Kim, S. S. Jang and S. G. Lee, RSC Adv., 2016, 6, 39587-39594.

13 X. H. Zheng, L. L. Song, R. N. Wang, H. Hao, L. J. Guo and Z. Zeng, Appl. Phys. Lett., 2010, 97, 666.

14 W. Y. Kim and K. S. Kim, Nat. Nanotechnol., 2008, 3, 408. 15 S. S. Alexandre, A. D. Lúcio, A. H. Neto and R. W. Nunes, Nano Lett., 2012, 12, 5097.

16 E. Loginova, S. Nie, K. Thürmer, N. C. Bartelt and K. F. Mccarty, Phys. Rev. B: Condens. Matter Mater. Phys., 2009, 80, 1956.

17 J. Lahiri, Y. Lin, P. Bozkurt, H. Oleynik and M. Batzill, Nat. Nanotechnol., 2010, 5, 326.

18 G. P. Tang, J. C. Zhou, Z. H. Zhang, X. Q. Deng and Z. Q. Fan, Carbon, 2013, 60, 94-101.

19 D. Wang, Z. H. Zhang, X. Q. Deng, Z. Q. Fan and G. P. Tang, Carbon, 2016, 98, 204-212.

20 M. Kiguchi, H. Nakamura, Y. Takahashi, T. Takahashi and T. Ohto, J. Phys. Chem. C, 2010, 114, 22254.

21 A. Saraivasouza, M. Smeu, H. Terrones, A. G. S. Filho and M. A. Ratner, J. Phys. Chem. C, 2015, 117, 21178-21185. 
22 C. M. Jaworski, R. C. Myers, E. Johnston-Halperin and J. P. Heremans, Nature, 2012, 487, 210.

23 K. R. Jeon, B. C. Min, A. Spiesser, H. Saito, S. C. Shin, S. Yuasa and R. Jansen, Nat. Mater., 2014, 13, 360.

24 S. G. Kwon, S. Back, J. E. Park and B. Kang, J. Mater. Chem. C, 2018, 6, 7759-7766.

25 R. Landauer, Philos. Mag., 1970, 21(172), 863.

26 J. Zeng and K. Q. Chen, J. Mater. Chem. C, 2013, 1, 40144019.

27 Y. Yamagishi, S. Nakashima, K. Oiso and K. Yamada, Nanotechnology, 2013, 24, 395704.

28 X. Q. Deng, Z. H. Zhang, L. Sun and L. J. Wu, Org. Electron., 2017, 41, 276-383.
29 M. Zeng, L. Shen, M. Zhou, C. Zhang and Y. Feng, Phys. Rev. B: Condens. Matter Mater. Phys., 2011, 83, 115427.

30 H. Q. Wan, B. H. Zhou, W. H. Liao and G. H. Zhou, J. Chem. Phys., 2013, 138, 034705.

31 X. Zhou, Y. Liu, M. Zhou, H. Shao and G. Zhou, Appl. Phys. Express, 2014, 7, 021201.

32 G. C. Solomon, C. Herrmann, T. Hansen, V. Mujica and M. A. Ratner, Nat. Chem., 2010, 2, 223.

33 D. Zhang, M. Long, X. Zhang and H. Xu, RSC Adv., 2015, 5, 96455.

34 Z. Q. Fan, W. Y. Sun, Z. H. Zhang, X. Q. Deng, G. P. Tang and H. Q. Xie, Carbon, 2017, 122, 687-693.

35 M. Zeng, Y. Feng and G. Liang, Nano Lett., 2011, 11, 1369. 